\title{
Brown adipose tissue thermogenesis in humans
}

\section{P. Nuutila}

Received: 20 June 2013 / Accepted: 26 June 2013 /Published online: 10 August 2013

(C) Springer-Verlag Berlin Heidelberg 2013

\begin{abstract}
The prevalence of obesity and type 2 diabetes is at epidemic proportions. Classical interventions aimed at targeting obesity, such as reducing energy intake or increasing exercise, are often not effective over the long term. In contrast to white adipocytes, which store energy, brown adipocytes generate heat via mitochondrial uncoupling protein 1 , thereby acting as a defence against hypothermia and, potentially, obesity. In this issue of Diabetologia, Admiraal et al compare brown adipose tissue activation during cold exposure between two different ethnic groups: South Asians and Europids. The prevalence of abdominal obesity and type 2 diabetes differs among various ethnic groups and decreased BAT metabolic activity could be one causal factor. As yet, the clinical impact of this 'rediscovered' organ is largely unknown, but has potential as a drug target for obesity.
\end{abstract}

Keywords Adipocyte - Brown adipose tissue .

$\left[{ }^{18} \mathrm{~F}\right]$ Fluorodeoxyglucose (FDG) $\cdot$ Insulin $\cdot$ Obesity .

Positron emission tomography $\cdot$ UCP-1

\section{Brown adipose tissue: its 'rediscovery'}

Brown adipose tissue (BAT) was first detected in small mammals and infants and defined as an adaptation to defend against the cold and to maintain body temperature Many researchers over the decades have thought that brown adipose tissue is also important in adult humans. More than 30 years ago, autopsy studies showed brown adipocytes around the neck arteries of adult outdoor workers [1], but the evidence of their functionality was missing. The role of these adipocytes remained unclear until the use of functional imaging modalities, including positron emission tomography (PET) with the glucose analogue $\left[{ }^{18} \mathrm{~F}\right]$ fluorodeoxyglucose (FDG) used as a tracer, became widely used in diagnostics and the association between the deposition of supraclavicular FDG and the cold outdoor temperature was reported [2, 3]. These FDG 'hot spots' were problematic for nuclear physicists because the images were taken from patients with oncological indications. When FDG PET imaging was combined with analysis of biopsies taken from metabolically active BAT depots [4-6], BAT emerged as a physiologically active tissue in adult humans. The several-fold increase in cold-induced glucose uptake in supraclavicular BAT depots suggested that, if fully activated, they have the potential to consume or burn an amount of energy equivalent to several kilograms of fat over the course of a year [4]. Over the past few years, this 're-discovery' of BAT has stimulated a rush for researchers to elucidate pathways that will trigger its development and metabolic activation.

Humans have two different types of BAT: 'classical BAT', found in the interscapular area in infants, and 'inducible thermogenic BAT', also known as beige or brite fat $[7,8]$. Image-guided biopsies of BAT depots, taken from adult humans, confirmed by morphology and high expression of UCP-1 [4], demonstrated that this depot consists of inducible brown adipocytes [8]. The molecular mechanisms underlying BAT activation and/or browning are still poorly understood. 
Several of the involved pathways and factors, such as irisin, have been discovered [9].

\section{Obesity, insulin resistance and BAT activity}

In rodents, thermogenesis, orchestrated by BAT, is important for weight maintenance during a high-fat diet, and in humans, cold-induced BAT activity is inversely associated with BMI $[5,10]$. In this issue of Diabetologia, Admiraal et al report the results of a study in which they compared BAT activation during cold-exposure between two different ethnic groups: South Asians and Europids [11]. In the UK, the risk of type 2 diabetes is four- to sixfold higher in the South-Asian population than in non-South-Asian populations [12]. This may be explained by multiple factors that contribute to disease pathogenesis, such as an increased susceptibility to the development of insulin resistance or the adoption of a sedentary lifestyle. Decreased BAT activity could also be a factor.

Admiraal et al recruited healthy young men with normal BMIs; ten Europid and ten South Asian [11]. BAT activity was measured after cold exposure using static FDG PETcomputed tomography scanning. Maximal standardised uptake values $\left(\mathrm{SUV}_{\max }\right)$ were used for the assessment of glucose uptake. This semiquantitative method gives useful information during cold exposure, because FDG uptake into skeletal muscle is low. The variation in $\mathrm{SUV}_{\max }$ values was high. A wide variation in values during cold exposure has also been found when FDG data have been modelled $[4,10]$. The main result of Admiraal et al was that the expected difference in BAT activity between ethnic groups was not found [11]. The low number of participants included in the study may be responsible for the lack of evidence supporting the hypothesis that BAT specifically contributes to weight gain in the South-Asian population.

\section{Brown adipose tissue and insulin sensitivity}

In line with previous reports [10,13], Admiraal et al [11] did not find any correlation between whole body insulin sensitivity measured using euglycaemic clamp and BAT activity. The mechanisms by which BAT influences insulin sensitivity are indirect and complex. In humans, BAT is highly insulin sensitive. Its glucose uptake is, on average, fivefold higher in response to insulin stimulation compared with fasting conditions, and similarly as insulin enhances skeletal muscle uptake per tissue weight [13]. Moreover, insulin stimulated glucose uptake rate in BAT correlates with whole body $M$ value, a measure of whole body insulin sensitivity [10]. As a result of its lighter total tissue weight, the contribution of BAT to whole body insulin sensitivity is minor.

The effects of insulin stimulation and mild cold exposure on BAT metabolism are different, although both produce a remarkable increase in glucose uptake rate in BAT and induce elevated energy expenditure [13]. Insulin increases glucose transport into BAT, influences its storage capacity and decreases the availability of fatty acids from the circulation. In contrast, the increased glucose uptake observed during acute cold exposure is perfusion-driven. Fatty acid uptake in BAT is also increased [14] and, as a result of sympathetic activation, plasma noradrenaline concentrations and NEFA levels are elevated and plasma insulin levels are markedly decreased [13]. Compared with lean individuals, obese insulin-resistant individuals have blunted or absent perfusion responses in BAT during cold [10]. Taken together, these results indicate that a direct association between insulin sensitivity and BAT activity is unlikely. During cold exposure, thermogenesis produced by BAT, estimated using tissue glucose uptake rate, is a dynamic, mostly neuronally mediated process.

\section{The impact of BAT on metabolism}

In rodents, BAT activity and browning of adipose tissue are crucial for thermogenesis and survival. In mice, BAT protects against diet-induced obesity, insulin resistance and type 2 diabetes. The role of BAT in humans is not fully understood. According to direct measurements using FDG-PET during cold stimulation, glucose metabolism is increased by tenfold in supraclavicular BAT depots, suggesting a significant increment in thermogenesis [4]. In line with this, Muzik and co-workers found increased oxygen consumption in activated BAT depots, but the magnitude of the increase was too low to be clinically significant [14]. However, assessment of oxygen consumption in BAT with ${ }^{15} \mathrm{O}_{2}$ PET has not been validated. The rapid oxygen kinetics and limited resolution of human PET scanners could lead to underestimated values in small fat depots.

The distribution of brown adipocytes in the human body is a special challenge for its research and understanding. Instead of more comprehensive analyses, human studies have focused on supraclavicular BAT depots. The plasticity of BAT might also suggest that even smaller BAT depots should be included into the analyses in order to accurately assess BAT activity. We need new methods to measure both BAT mass and metabolic activity. The quantification of BAT is demanding because BAT is a mixture of beige (brite) and white adipocytes. The combined PET-MRI scanner is a potentially optimal tool for further evaluation of BAT activation in clinical studies under both normal and stimulated conditions. 
In summary, advancing human brown fat metabolic analysis and quantitation with PET-MRI modalities will augment ongoing mechanistic studies that could unlock breakthroughs in obesity research.

Duality of interest The author declares that there is no duality of interest associated with this manuscript.

Contribution statement The author was the sole contributor to this paper.

\section{References}

1. Huttunen P, Hirvonen J, Kinnula V (1981) The occurrence of brown adipose tissue in outdoor workers. Eur J Appl Physiol 46:339-345

2. Cohade C, Mourtzikos KA, Wahl RL (2003) "USA-Fat": prevalence is related to ambient outdoor temperature - evaluation with ${ }^{18}$ F-FDG PET/CT. J Nucl Med 44:1267-1270

3. Persichetti A, Sciuto R, Rea S et al (2013) Prevalence, mass, and glucose-uptake activity of ${ }^{18} \mathrm{~F}$-FDG-detected brown adipose tissue in humans living in a temperate zone of Italy. PLoS One 8:e63391

4. Virtanen KA, Lidell ME, Orava J et al (2009) Functional brown adipose tissue in healthy adults. N Engl J Med 360:1518-1525
5. van Marken Lichtenbelt WD, Vanhommerig JW, Smulders NM et al (2009) Cold-activated brown adipose tissue in healthy men. N Engl J Med 360:1500-1508

6. Cypress AM, Lehman S, Williams G et al (2009) Identification and importance of brown adipose tissue in adult humans. N Engl J Med 360:1509-1517

7. Wu J, Boström P, Sparks LM et al (2012) Beige adipocytes are a distinct type of thermogenic fat cell in mouse and human. Cell 150:366-376

8. Lidell ME, Betz MJ, Leinhard OD et al (2013) Evidence for two types of brown adipose tissue in humans. Nat Med 19:631-634

9. Spiegelman BM (2013) 2012 Banting Lecture: regulation of adipogenesis: toward new therapeutics for metabolic disease. Diabetes 62:1774-1782

10. Orava J, Nuutila P, Noponen T et al (2013) Blunted metabolic responses to cold and insulin stimulation in brown adipose tissue of obese humans. Obesity. doi:10.1002/oby.20456

11. Admiraal WM, Verberne HJ, Karamat FA, Soeters MR, Hoekstra JBL, Holleman F (2013) Cold-induced activity of brown adipose tissue in young lean men of South-Asian and European origin. Diabetologia. doi:10.1007/s00125-013-2938-5

12. Barnett AH, Dixon AN, Bellary S et al (2006) Type 2 diabetes and cardiovascular risk in the UK south Asian community. Diabetologia 10:2234-2246

13. Orava J, Nuutila P, Lidell ME et al (2011) Different metabolic responses of human brown adipose tissue to activation by cold and insulin. Cell Metab 14:272-279

14. Muzik O, Mangner TJ, Granneman JG (2012) Assessment of oxidative metabolism in brown fat using PET imaging. Front Endocrinol (Lausanne) 3:15 